\title{
A STRENGTHENED CAUCHY-SCHWARZ INEQUALITY FOR BIORTHOGONAL WAVELETS
}

\author{
ALESSANDRA DE Rossi
}

\begin{abstract}
A strengthened Cauchy-Schwarz inequality for spaces of biorthogonal wavelets defined on the real line and on the interval is proved. The strengthened Cauchy-Schwarz inequality is a fundamental tool in the analysis of the multilevel methods and, in particular, plays an important role in the a posteriori error estimates for hierarchical methods.
\end{abstract}

Mathematics subject classification (1991): 42C99.

Key words and phrases: biorthogonal wavelets, strengthened Cauchy-Schwarz inequality.

\section{REFERENCES}

[AHJP] L. Anderson, N. Hall, B. Jawerth, G. Peters, Wavelets on closed subsets of the real line, in Recent advances in wavelet analysis, L.L.Schumaker, G.Webb (eds.), Academic Press, 1991, $1-61$.

[BB] R. E. BANK, M. BENBOURENANE, The hierarchical basis multigrid method for convection-diffusion equations, Numer. Math., 61 (1992), 7-37.

[BDY] R. E. BANK, T. F. DUPONT, H. YSERENTANT, The hierarchical basis multigrid method, Numer. Math., 52 (1988), 427-458.

[BS] R. E. BANK, R. K. SMITH, A posteriori error estimates based on hierarchical bases, SIAM J. Numer. Anal., 30 (1993), 921-935.

[CC] C. CANUTO, I. CRAVERO, A wavelet-based adaptive finite element method for advection-diffusion equations, $M^{3} A S, 7$ (1997), 265-289.

[CT] C. CANUTO, A. TABACCO, Multilevel decompositions of functional spaces, J. Fourier Anal. and Appl., 3 (1997), 715-742.

[C] C. K. CHU, An introduction to wavelets, Academic Press, San Diego, 1992.

[CDF] A. COHEN, I. DAUBEChIES, J. C. FEAUVEAU, Biorthogonal bases of compactly supported wavelets, Comm. Pure Appl. Math., XLV (1992), 485-560.

[CDV] A. COHEN, I. DAUBECHIES, P. VIAL, Wavelets on the interval and fast wavelet transform, AT \& T Bell Laboratories, preprint (1992).

[CDJV] A. COhEn, I. Daubechies, B. Jawerth, P. Vial, Multiresolution analysis, wavelets and fast algorithms on an interval, C. R. Acad. Sci. Paris, 316 (1993), 417-421.

[Dah] W. DAHMEN, Multiscale analysis, approximation, and interpolation space, in Approximation Theory VIII, C. K. Chui, L. L. Schumaker (eds.), (1995), 1-41.

[DKU] W. Dahmen, A. Kunoth, K. URban, Biorthogonal Spline Wavelets on the Interval - Stability and Moment Conditions, RWTH Aachen, 1996.

[Dau] I. DAUBECHIES, Ten lectures on wavelets, CBMS-NSF Series in Applied Mathematics 61, SIAM, Philadelphia, 1992.

[De1] A. De RossI, Tesi di Dottorato, Università di Milano (1997).

[De2] A. DE RossI, A posteriori error estimates for hierarchical methods, preprint, Università di Torino, 3 (1997).

[EV] V. EiJkhout, P. VAssilevski, The role of the strengthened Cauchy-Buniakowskii-Schwarz inequality in multilevel methods, SIAM Review, 33 (1991), 405-419. 
[MT] M. MARION, R. TEMAM, Nonlinear Galerkin methods: the finite elements case, Numer. Math., 57 (1990), 205-226.

[M] Y. MEYER, Ondelettes et operatéurs I, Hermann, Paris, 1990.

[Y] H. YSERENTANT, On the multi-level splitting of finite element spaces, Numer. Math., 49 (1986), $379-412$. 